Article

\title{
Screening and Application of Chitin Synthase Inhibitors
}

\author{
Xiaozai Shi ${ }^{\circ}$, Shuo Qiu, Yingling Bao, Hanchi Chen, Yuele Lu and Xiaolong Chen* \\ College of Biotechnology and Bioengineering, Zhejiang University of Technology, Hangzhou 310014, China; \\ 18989480172@163.com (X.S.); zjutqs3@163.com (S.Q.); yinglingbao@zjut.edu.cn (Y.B.); \\ hchen23@zjut.edu.cn (H.C.); luyuele@zjut.edu.cn (Y.L.) \\ * Correspondence: Richard_chen@zjut.edu.cn
}

Received: 21 July 2020; Accepted: 20 August 2020; Published: 22 August 2020

\begin{abstract}
Chitin is an important part of the fungal cell wall, but is not found in plants and mammals, so chitin synthase (CHS) can be a green fungicide target. In this paper, 35 maleimide compounds were designed and synthesized as CHS inhibitors. All the screened compounds showed different degrees of CHS inhibitory activity and antifungal activity in vitro. In particular, the half-inhibitory concentration $\left(\mathrm{IC}_{50}\right)$ value of compound 20 on CHS was $0.12 \mathrm{mM}$, and the inhibitory effect was better than that of the control polyoxin $\mathrm{B}\left(\mathrm{IC}_{50}=0.19 \mathrm{mM}\right)$. At the same time, this compound also showed good antifungal activity and has further development value.
\end{abstract}

Keywords: chitin synthase; maleimides; inhibitors; biological activity research

\section{Introduction}

Fungal infections are a worldwide threat to agricultural economy and human health [1,2]. However, effective and safe antifungal drugs are very limited, and drug resistance risks are on the rise. Therefore, there is a substantial and constant need for novel antifungal agents [3].

Chitin, a polymer of $N$-acetylglucosamine conjugated by $\beta-1,4$ glycoside bonds, is an essential structural polysaccharide found in all fungal cell walls but not in vertebrates and plants [4]. Thus, CHS, the key enzyme in charge of catalyzing chitin biosynthesis, is an attractive and green target for new fungicides [5]. The earliest research on CHS inhibitors dates back to the late 1950s. Isono et al. isolated and extracted polyoxin from streptomycin, and found that it can inhibit the growth and reproduction of fungi by reducing the synthesis of chitin [6]. Since then, researchers have successively screened and obtained a variety of compounds that have a mild inhibitory effect on CHS by separation from natural products, extraction from plants, or chemical synthesis.

In the 1980s, Japanese scientists Kobiata et al. isolated new polyoxins (A, B and C) from Streptomyces cacaoi, which can be widely used in agricultural fungicides to control plant pathogens $[7,8]$. Nikkomycin, which was isolated from Streptomyces tendae and had a structure similar to polyoxin, also had great potential as an agricultural insecticide and fungicide. It is also the only CHS inhibitor that has entered clinical trials so far to treat fungal infections [9]. In 1999, Korean scientists Kim et al. isolated and extracted ursolic acid from the leaves of hawthorn. They found that it can be used as a selective inhibitor of chitin synthase II (CHS II), which is a branch of CHS, and its main function is to form a membrane. The $\mathrm{IC}_{50}$ towards Saccharomyces cerevisiae can reach $0.184 \mu \mathrm{g} / \mathrm{mL}$. In the same year, they isolated and extracted gosin $\mathrm{N}$ and wuwezisu $\mathrm{C}$ from Schisandra chinensis that had excellent inhibitory effects on CHS II, and the inhibitory effect was much better than polyoxin $\mathrm{D}\left(\mathrm{IC}_{50}\right.$ values of $6214 \mu \mathrm{g} / \mathrm{mL}$ and $1912 \mu \mathrm{g} / \mathrm{mL}$, respectively). In 2002, the research team once again isolated and extracted obovatol from Magnolia officinalis, which was a competitive inhibitor of CHS II, and its derivative tetrahydrobovatol also showed strong inhibitory activity against CHS II, with an $\mathrm{IC}_{50}$ of up 
to $59 \mu \mathrm{g} / \mathrm{mL}[10,11]$. Japanese scientists Obi et al. designed a series of compounds by introducing a hydrophobic group at the $\beta$ position of the amino acid chain of nikkomycin $\mathrm{Z}$ to replace the $\beta-\mathrm{CH}_{3}$ and $\gamma-\mathrm{OH}$ groups of nikkomycin Z, they also found that the inhibitory activity of the compound KFC-431, which contains a phenanthrene structure, was no less than that of nikkomycin $Z$ through biological activity studies [12].

However, as scholars continue their in-depth research, these CHS inhibitors have also exposed some shortcomings such as poor chemical stability, a narrow antibacterial spectrum and high cost [13]. Therefore, it is of great significance to study new and highly effective CHS inhibitors.

Maleimide compounds a are five-membered heterocyclic compounds containing N (Figure 1). These compounds contain $\alpha, \beta$ unsaturated carbon-carbon double bonds, and have strong selectivity to various nucleophilic substances. This also determines that these compounds have a variety of biological activities, such as antibacterial [14], antifungal [15], insecticidal [16], anti-cancer and as an enzyme inhibitor $[17,18]$. Hwang and Zacchino et al. have reported that this class of compounds and their analogs have a good inhibitory effect on CHS. In particular, compounds b and c (Figure 1) have a higher inhibitory activity on CHS than that of nikkomycin, and the inhibitory activity of compounds $\mathrm{d}$ and e (Figure 1) on CHS were comparable to that of nikkomycin [19,20]. Previous studies have also shown that some maleimide compounds and their analogs had good inhibitory effects on Sclerotinia sclerotiorum and Botrytis cinereal [21-23].<smiles>[R]C1=C([R])C(=O)N([R])C1=O</smiles>

a<smiles>[R][X]c1cc2c(c(-c3ccccc3)c1C#N)C(=O)CCC2</smiles><smiles>COC1=C(OC)C(=O)C(=C([O-])C=Cc2ccccc2)C1=O</smiles>

b<smiles>[R]=C1C(=O)C(=O)N(C)C1=O</smiles>

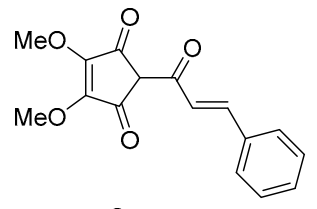

C

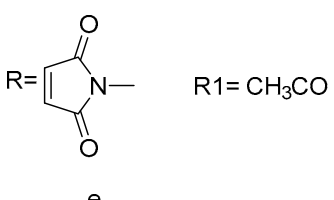

Figure 1. General formula of maleimide compounds (a), structures of methyllinderone (b), linderone (c), maleimide derivative $(\mathbf{d})$ and maleimide derivative $(\mathbf{e})$.

Recently, we have synthesized a series of maleimide compounds with excellent antifungal activity [24]. In order to screen out highly active maleimide compounds and use them as CHS inhibitors, based on previous research, we put more activity substituents in place to structure the maleimide ring. Thirty-five maleimide derivatives were synthesized with some modifications and their antifungal activity and CHS inhibitory effect were studied. All compound structures were characterized by ${ }^{1} \mathrm{H}$ NMR and high-resolution mass spectrometry (HRMS).

\section{Materials and Methods}

\subsection{Materials and Instruments}

All reactions were performed with commercially available reagents. S. sclerotiorum was isolated from sclerotia collected from a diseased plant of oilseed rape in Zhejiang, China, as described before [21]. The culture medium was potato dextrose agar (PDA). ${ }^{1} \mathrm{H}$ NMR spectra was recorded on a Bruker Advance I $500 \mathrm{MHz}$ spectrometer in dimethyl sulfoxide (DMSO) or dehydrated chloroform $\left(\mathrm{CDCl}_{3}\right)$ solutions using tetramethylsilane (TMS) as an internal standard. HRMS was determined by a Thermo LCQ/ADVANTAGE mass spectrometer (Thermo Fisher Scientific, Waltham, MA, USA); the CHS 
inhibition test was determined by an Elx808 (Bio Tek Instruments, Inc., Vermont, VT, USA) type microplate reader.

\subsection{Chemistry}

We designed, synthesized and evaluated a series of maleimide compounds (Figure 2), which were modified in both their five-membered ring structure and substituents on the benzene ring. All reactions are shown in Schemes 1 and 2. All target compounds are summarized in Table 1.<smiles>[R]C1C(=O)N(C)C(=O)C1[R]</smiles>

(f)<smiles>[R]C1C(=O)N(c2ccccc2)C(=O)C1[2H]</smiles>

(g)

Figure 2. The designed compounds 1-20 (f), the designed compounds 21-35 (g), n represented the number of the methylene groups, $R_{1}$ and $R_{2}$ represented the substituents on the five-membered ring, $R$ represented the substituents on the benzene ring.<smiles>[R]c1c([R])c(=O)[cH+]c1=O</smiles>

Scheme 1. Synthesis of compounds 1-20. Reagents and conditions: (i) acetone, $25^{\circ} \mathrm{C}, 1 \mathrm{~h}$. (ii) acetic anhydride, sodium acetate, $120^{\circ} \mathrm{C}, 3 \mathrm{~h}$.<smiles>[R][R]1ccc(N2C(=O)C([R])=C(C(=O)Nc3ccc([R])cc3)C2=O)cc1</smiles>

Scheme 2. Synthesis of compounds 21-35. Reagents and conditions: (i) acetic acid, $25{ }^{\circ} \mathrm{C}, 0.5 \mathrm{~h}$. (ii) $120^{\circ} \mathrm{C}, 3 \mathrm{~h}$.

Table 1. Maleimide compound structures.

\begin{tabular}{|c|c|c|c|}
\hline Compound & $\mathbf{n}$ & $\mathbf{R}_{1}$ & $\mathbf{R}_{\mathbf{2}}$ \\
\hline 1 & 0 & $\mathrm{H}$ & $\mathrm{H}$ \\
\hline 2 & 0 & $\mathrm{H}$ & $\mathrm{CH}_{3}$ \\
\hline 3 & 0 & $\mathrm{H}$ & Phenyl \\
\hline 4 & 0 & $\mathrm{CH}_{3}$ & $\mathrm{CH}_{3}$ \\
\hline 5 & 0 & $\mathrm{Cl}$ & $\mathrm{Cl}$ \\
\hline 6 & 1 & $\mathrm{H}$ & $\mathrm{H}$ \\
\hline 7 & 1 & $\mathrm{H}$ & $\mathrm{CH}_{3}$ \\
\hline 8 & 1 & $\mathrm{H}$ & Phenyl \\
\hline 9 & 1 & $\mathrm{CH}_{3}$ & $\mathrm{CH}_{3}$ \\
\hline 10 & 1 & $\mathrm{Cl}$ & $\mathrm{Cl}$ \\
\hline 11 & 2 & $\mathrm{H}$ & $\mathrm{H}$ \\
\hline 12 & 2 & $\mathrm{H}$ & $\mathrm{CH}_{3}$ \\
\hline 13 & 2 & $\mathrm{H}$ & Phenyl \\
\hline 14 & 2 & $\mathrm{CH}_{3}$ & $\mathrm{CH}_{3}$ \\
\hline 15 & 2 & $\mathrm{Cl}$ & $\mathrm{Cl}$ \\
\hline 16 & 3 & $\mathrm{H}$ & $\mathrm{H}$ \\
\hline 17 & 3 & $\mathrm{H}$ & $\mathrm{CH}_{3}$ \\
\hline 18 & 3 & $\mathrm{H}$ & Phenyl \\
\hline 19 & 3 & $\mathrm{CH}_{3}$ & $\mathrm{CH}_{3}$ \\
\hline 20 & 3 & $\mathrm{Cl}$ & $\mathrm{Cl}$ \\
\hline
\end{tabular}


Table 1. Cont.

\begin{tabular}{|c|c|c|c|}
\hline Compound & $\mathbf{R}$ & $\mathbf{R}_{1}$ & $\mathbf{R}_{\mathbf{2}}$ \\
\hline 21 & $\mathrm{~F}$ & $\mathrm{H}$ & $\mathrm{H}$ \\
\hline 22 & $\mathrm{~F}$ & $\mathrm{H}$ & $\mathrm{CH}_{3}$ \\
\hline 23 & $\mathrm{~F}$ & $\mathrm{H}$ & Phenyl \\
\hline 24 & $\mathrm{~F}$ & $\mathrm{CH}_{3}$ & $\mathrm{CH}_{3}$ \\
\hline 25 & $\mathrm{~F}$ & $\mathrm{Cl}$ & $\mathrm{Cl}$ \\
\hline 26 & $\mathrm{Cl}$ & $\mathrm{H}$ & $\mathrm{H}$ \\
\hline 27 & $\mathrm{Cl}$ & $\mathrm{H}$ & $\mathrm{CH}_{3}$ \\
\hline 28 & $\mathrm{Cl}$ & $\mathrm{H}$ & Phenyl \\
\hline 29 & $\mathrm{Cl}$ & $\mathrm{CH}_{3}$ & $\mathrm{CH}_{3}$ \\
\hline 30 & $\mathrm{Cl}$ & $\mathrm{Cl}$ & $\mathrm{Cl}$ \\
\hline 31 & $\mathrm{Br}$ & $\mathrm{H}$ & $\mathrm{H}$ \\
\hline 32 & $\mathrm{Br}$ & $\mathrm{H}$ & $\mathrm{CH}_{3}$ \\
\hline 33 & $\mathrm{Br}$ & $\mathrm{H}$ & Phenyl \\
\hline 34 & $\mathrm{Br}$ & $\mathrm{CH}_{3}$ & $\mathrm{CH}_{3}$ \\
\hline 35 & $\mathrm{Br}$ & $\mathrm{Cl}$ & $\mathrm{Cl}$ \\
\hline
\end{tabular}

\subsection{Biological Activity Assay}

\subsubsection{Inhibition on Chitin Synthase Assay}

The CHS inhibition assay was performed using $S$. sclerotiorum yeast cell extracts according to the method with some modifications described by Ke et al. [25]. In brief, S. sclerotiorum mycelium were inoculated into $250 \mathrm{~mL}$ PDA liquid medium from fresh S. sclerotiorum PDA solid medium, and cultured at $23{ }^{\circ} \mathrm{C}$ for $36 \mathrm{~h}$. The fungal cells were collected by centrifugation at $3000 \times g$ for $10 \mathrm{~min}$. Then, the harvested cells were disrupted in liquid nitrogen after being washed twice with ultrapure water. After digestion by $80 \mu \mathrm{g} / \mathrm{mL}$ trypsin at $30^{\circ} \mathrm{C}$ for $30 \mathrm{~min}, 120 \mu \mathrm{g} / \mathrm{mL}$ soybean trypsin inhibitor was added to terminate the reaction. Finally, we collected the supernatant to obtain the crude CHS solution at $3000 \mathrm{~g}$ for $10 \mathrm{~min}$, and stored it at $-20^{\circ} \mathrm{C}$.

CHS activity was measured on a 96-well microtiter plate coated with wheat germ agglutinin (WGA). All test samples were formulated into a drug solution with a concentration of $10 \mathrm{mg} / \mathrm{mL}$ with DMSO and diluted to $300,150,75,37.5,18.75,9.375$ and $4.69 \mu \mathrm{g} / \mathrm{mL}$ with $50 \mathrm{mM}$ Tris- $\mathrm{HCl}$ buffer, respectively. Trypsin pretreated cell extracts $(48 \mu \mathrm{L}), 50 \mu \mathrm{L}$ premixed solution $\left(3.2 \mathrm{mM} \mathrm{CoCl}_{2}, 80 \mathrm{mM} \mathrm{GlcNAc}, 8 \mathrm{mM}\right.$ UDP-GlcNAc dissolved in $50 \mathrm{mM}$ Tris-HCl buffer $\mathrm{pH}$ 7.5) and $2 \mu \mathrm{L}$ of test sample or DMSO (control) were added to each well. The plate was immediately washed 6 times with ultrapure water after it was incubated on a shaker at $30^{\circ} \mathrm{C}$ for $3 \mathrm{~h}$. Then, $100 \mu \mathrm{L}$ of horseradish peroxidase conjugated wheat germ agglutinin (WGA-HRP) solution (1 mg/mL WGA-HRP and $20 \mathrm{mg} / \mathrm{mL}$ bovine serum albumin (BSA) dissolved in $50 \mathrm{mM}$ Tris- $\mathrm{HCl}$ buffer $\mathrm{pH}$ 7.5) was added to each well. The plate was washed 6 times with ultrapure water after it was incubated at $30^{\circ} \mathrm{C}$ for $30 \mathrm{~min}$. Finally, $100 \mu \mathrm{L}$ fresh tetramethylbenzidine (TMB) reaction solution $\left(0.8 \mathrm{mmol} / \mathrm{L} \mathrm{TMB}\right.$ and $1.8 \% \mathrm{H}_{2} \mathrm{O}_{2}$ dissolved in $100 \mathrm{mmol} / \mathrm{L}$ sodium acetate buffer, $\mathrm{pH}$ 3.7) was added to it. The optical density (OD) values at $600 \mathrm{~nm}$ were detected every $2 \mathrm{~min}$ for $40 \mathrm{~min}$. The reaction rate was calculated based on the measured $\mathrm{OD}_{600}$ values. The $\mathrm{IC}_{50}$ values were calculated by using the reaction rate derived from 7 different compound concentrations.

\subsubsection{Antifungal Assays}

Antifungal activities and structure-activity relationship studies against S. sclerotiorum were previously investigated in vitro by the mycelial growth rate method [26]. All test samples were dissolved in $0.1 \%$ Tween- 80 containing $10 \%$ acetone and formulated into drug-containing PDA medium at final concentrations of 50, 25, 12.5, 6.25, 3.125, and $1.56 \mu \mathrm{g} / \mathrm{mL}$. Each concentration gradient set two parallels, and $1.0 \mathrm{~mL}$ of $0.1 \%$ Tween- 80 containing $10 \%$ acetone plus $9.0 \mathrm{~mL}$ of sterilized and melted PDA medium was used as the blank control. These dishes were incubated at $23^{\circ} \mathrm{C}$ for $36 \mathrm{~h}$, 
and then the diameter of each colony was measured by making two measurements at right angles. The inhibition rate was calculated using the following formula:

$$
\text { Inhibition of growth }(\%)=\left(D_{c k}-D\right) /\left(D_{c k}-6\right) \times 100 \text {, }
$$

where $\mathrm{D}_{\mathrm{ck}}$ is the average colony diameter of the blank control, $\mathrm{D}$ is the average colony diameter of test compounds, and 6 is the diameter of the inoculum plug (in $\mathrm{mm}$ ). With a logit-log transformation, the concentration-response curves were linearized by regression, and then the half-effective concentration $\left(\mathrm{EC}_{50}\right)$ values of different compounds on mycelial growth was calculated [27].

\section{Results}

\subsection{General Synthetic Procedure for Maleimides 1-20}

In the first step, maleic anhydride $(1.96 \mathrm{~g}, 0.02 \mathrm{~mol})$ and aniline $(1.82 \mathrm{~mL}, 0.02 \mathrm{~mol})$ were added to a three-necked flask equipped with a magnetic stirrer, then $15 \mathrm{~mL}$ of acetone solution was added at room temperature for $1 \mathrm{~h}$ to generate the corresponding maleamic acid. For the second step, $5 \mathrm{~mL}$ acetic anhydride and $100 \mathrm{mg}$ sodium acetate were added to the three-necked flask and then the reaction mixture was heated to $120^{\circ} \mathrm{C}$ and refluxed for 2-3 h. Finally, the concentrated solution was obtained by rotary evaporation after the reaction solution was cooled to room temperature. The concentrated solution was isolated by silica gel column to obtain the target compound $\mathbf{1}$. Other maleimide derivatives were synthesized similarly. The physical constants and spectral data of compounds 1-20 are summarized below.

\subsubsection{N-phenyl-maleimide (1)}

Yield 78.6\%. ${ }^{1} \mathrm{H}$ NMR (500 MHz, $\left.\mathrm{CDCl}_{3}\right)$ 87.38-7.35 (m, 2H), 7.35-7.31 (m, 2H), $7.29(\mathrm{dq}, \mathrm{J}=3.9$, $1.7 \mathrm{~Hz}, 1 \mathrm{H}), 6.72(\mathrm{~d}, \mathrm{~J}=5.0 \mathrm{~Hz}, 2 \mathrm{H})$. HRMS [M + H] $]^{+}: 174.1715$, found, 174.0477.

\subsubsection{N-phenyl-3-methylmaleimide (2)}

Yield 72.3\%. ${ }^{1} \mathrm{H}$ NMR $\left(500 \mathrm{MHz}, \mathrm{CDCl}_{3}\right) \delta 7.47(\mathrm{dt}, \mathrm{J}=9.9,1.9 \mathrm{~Hz}, 2 \mathrm{H}), 7.37(\mathrm{tt}, \mathrm{J}=3.6,1.7 \mathrm{~Hz}$, 2H), 7.36-7.34 (m, 1H), $6.05(\mathrm{q}, \mathrm{J}=1.8 \mathrm{~Hz}, 1 \mathrm{H}), 2.19(\mathrm{t}, \mathrm{J}=2.2 \mathrm{~Hz}, 3 \mathrm{H})$. HRMS $[\mathrm{M}+\mathrm{H}]^{+}: 188.0645$, found, 188.0633 .

\subsubsection{N-phenyl-3-phenylmaleimide (3)}

Yield 83.3\%. ${ }^{1} \mathrm{H}$ NMR (500 MHz, $\left.\mathrm{CDCl}_{3}\right) \delta 7.67(\mathrm{~m}, 5 \mathrm{H}), 7.55(\mathrm{~m}, 1 \mathrm{H}), 7.47(\mathrm{~m}, 4 \mathrm{H}), 7.27(\mathrm{~s}, 1 \mathrm{H})$. HRMS $[\mathrm{M}+\mathrm{H}]^{+}: 250.0788$, found, 250.0790 .

\subsubsection{N-phenyl-3,4-dimethylmaleimide (4)}

Yield 79.3\%. ${ }^{1} \mathrm{H}$ NMR (500 MHz, DMSO) $87.50(\mathrm{~d}, \mathrm{~J}=7.9 \mathrm{~Hz}, 2 \mathrm{H}), 7.32-7.28(\mathrm{~m}, 2 \mathrm{H}), 7.02(\mathrm{t}, \mathrm{J}=7.4$ $\mathrm{Hz}, 1 \mathrm{H}), 2.07(\mathrm{~s}, 6 \mathrm{H})$. HRMS [M + H] $]^{+}: 202.0788$, found, 202.0790 .

\subsubsection{N-phenyl-3,4-dichloromaleimide (5)}

Yield 70.3\%. ${ }^{1} \mathrm{H}$ NMR (500 MHz, $\left.\mathrm{CDCl}_{3}\right) \delta 7.53-7.48(\mathrm{~m}, 2 \mathrm{H}), 7.43(\mathrm{dd}, \mathrm{J}=8.5,6.4 \mathrm{~Hz}, 1 \mathrm{H})$, 7.38-7.33 (m, 2H). HRMS [M + H] $]^{+}$241.9686, found, 241.9697.

\subsection{6. $N$-benzyl-maleimide (6)}

Yield 73.3\%. ${ }^{1} \mathrm{H}$ NMR (500 MHz, $\left.\mathrm{CDCl}_{3}\right)$ 87.38-7.35 (m, 2H), 7.35-7.31 (m, 2H), 7.29 (dq, J = 3.9, $1.7 \mathrm{~Hz}, 1 \mathrm{H}), 6.72(\mathrm{~d}, \mathrm{~J}=5.0 \mathrm{~Hz}, 2 \mathrm{H}), 4.69(\mathrm{~s}, 2 \mathrm{H})$. HRMS [M + H] ${ }^{+}:$188.1985, found, 188.1980. 
3.1.7. N-benzyl-3-methylmaleimide (7)

Yield 76.2\%. ${ }^{1} \mathrm{H}$ NMR (500 MHz, $\left.\mathrm{CDCl}_{3}\right)$ 87.38-7.34 (m, 2H), 7.34-7.30 (m, 2H), 7.30-7.26 (m, 1H), $6.34(\mathrm{q}, \mathrm{J}=1.8 \mathrm{~Hz}, 1 \mathrm{H}), 4.67(\mathrm{~s}, 2 \mathrm{H}), 2.09(\mathrm{~d}, \mathrm{~J}=1.8 \mathrm{~Hz}, 3 \mathrm{H})$. HRMS [M + H] $]^{+}: 202.0775$, found, 202.0790 .

3.1.8. N-benzyl-3-phenylmaleimide (8)

Yield 73.7\%. ${ }^{1} \mathrm{H}$ NMR $\left(500 \mathrm{MHz}, \mathrm{CDCl}_{3}\right) \delta 7.85-7.65(\mathrm{~m}, 6 \mathrm{H}), 7.58(\mathrm{dt}, \mathrm{J}=4.7,2.7 \mathrm{~Hz}, 2 \mathrm{H})$, 7.47-7.45(m, 1H), 7.39-7.36 (m, 2H), 4,74 (m, 2H). HRMS [M + H] ${ }^{+}:$264.0953, found, 264.0946.

3.1.9. N-benzyl-3,4-dimethylmaleimide (9)

Yield 78.2\%. ${ }^{1} \mathrm{H}$ NMR (500 MHz, $\left.\mathrm{CDCl}_{3}\right) \delta 7.37-7.30(\mathrm{~m}, 5 \mathrm{H}), 4.66(\mathrm{~s}, 2 \mathrm{H}), 1.97$ (s, 6H). HRMS $[\mathrm{M}+\mathrm{H}]^{+}:$216.0954, found, 216.0946 .

3.1.10. N-benzyl-3,4-dichloromaleimide (10)

Yield 72.7\%. ${ }^{1} \mathrm{H}$ NMR (500 MHz, $\left.\mathrm{CDCl}_{3}\right) \delta 7.38(\mathrm{dt}, \mathrm{J}=4.7,2.7 \mathrm{~Hz}, 2 \mathrm{H}), 7.37-7.35(\mathrm{~m}, 1 \mathrm{H})$, 7.35-7.30 (m, 2H), $4.76(\mathrm{~s}, 2 \mathrm{H})$. HRMS [M + H] ${ }^{+}: 255.9848$, found, 255.9854.

3.1.11. $\mathrm{N}$-phenethyl-maleimide (11)

Yield 77.2\%. ${ }^{1} \mathrm{H}$ NMR $\left(500 \mathrm{MHz}, \mathrm{CDCl}_{3}\right) \delta 7.50-7.46(\mathrm{~m}, 2 \mathrm{H}), 7.29-7.24(\mathrm{~m}, 2 \mathrm{H}), 7.24-7.18(\mathrm{~m}, 2 \mathrm{H})$, $7.16(\mathrm{~s}, 1 \mathrm{H}), 3.69-3.65(\mathrm{~m}, 2 \mathrm{H}), 2.86-2.82(\mathrm{~m}, 2 \mathrm{H})$. HRMS [M + H] ${ }^{+}:$202.0785, found, 202.0790.

3.1.12. N-phenethyl-3-methylmaleimide (12)

Yield 70.2\%. ${ }^{1} \mathrm{H}$ NMR (500 MHz, $\left.\mathrm{CDCl}_{3}\right)$ 87.29-7.24 (m, 2H), 7.24-7.18 (m, 2H), 7.16 (s, 1H), $6.92(\mathrm{~s}, 1 \mathrm{H}), 3.75(\mathrm{~s}, 1 \mathrm{H}), 3.60(\mathrm{~s}, 1 \mathrm{H}), 2.86-2.82(\mathrm{~m}, 2 \mathrm{H}), 1.91-1.87(\mathrm{~m}, 3 \mathrm{H}) . \mathrm{HRMS}[\mathrm{M}+\mathrm{H}]^{+}:$216.0952, found, 216.0946.

3.1.13. $N$-phenethyl-3-phenylmaleimide (13)

Yield 76.9\%. ${ }^{1} \mathrm{H}$ NMR (500 MHz, $\left.\mathrm{CDCl}_{3}\right) \delta 7.50-7.41(\mathrm{~m}, 3 \mathrm{H}), 7.41-7.36(\mathrm{~m}, 2 \mathrm{H}), 7.31(\mathrm{~s}, 1 \mathrm{H})$, 7.23-7.18 (m, 2H), 7.14-7.05 (m, 3H), $3.85(\mathrm{~s}, 1 \mathrm{H}), 3.67(\mathrm{~s}, 1 \mathrm{H}), 2.88-2.84(\mathrm{~m}, 2 \mathrm{H}) . \mathrm{HRMS}[\mathrm{M}+\mathrm{H}]^{+}$: 278.1112, found, 278.1103 .

3.1.14. N-phenethyl-3,4-dimethylmaleimide (14)

Yield 67.3\%. ${ }^{1} \mathrm{H}$ NMR (500 MHz, $\left.\mathrm{CDCl}_{3}\right) \delta 7.29-7.24(\mathrm{~m}, 2 \mathrm{H}), 7.24-7.18(\mathrm{~m}, 2 \mathrm{H}), 7.16(\mathrm{~s}, 1 \mathrm{H})$, 3.70-3.66 (m, 2H), 2.86-2.82 (m, 2H), 1.90-1.86 (m, 6H). HRMS [M + H] $]^{+}: 230.1112$, found, 230.1103.

3.1.15. N-phenethyl-3,4-dichloromaleimide (15)

Yield 73.8\%. ${ }^{1} \mathrm{H}$ NMR (500 MHz, $\left.\mathrm{CDCl}_{3}\right) \delta$ 7.29-7.26 (m, 2H), 7.26-7.13 (m, 6H), 3.70-3.66 (m, 3H), 2.85-2.81 (m, 3H). HRMS [M + H] : 270.0016, found, 270.0010.

3.1.16. $\mathrm{N}$-phenylpropyl-maleimide (16)

Yield 73.5\%. ${ }^{1} \mathrm{H}$ NMR $\left(500 \mathrm{MHz}, \mathrm{CDCl}_{3}\right)$ 87.48-7.44 (m, 1H), 7.29-7.22 (m, 1H), 7.22-7.11 (m, 2H), 3.42-3.38 (m, 1H), 2.62-2.58 (m, 1H), 1.93-1.89 (m, 1H). HRMS [M + H] $]^{+}: 216.0958$, found, 216.0946.

3.1.17. $\mathrm{N}$-phenylpropyl-3-methylmaleimide (17)

Yield 66.5\%. ${ }^{1} \mathrm{H}$ NMR (500 MHz, $\left.\mathrm{CDCl}_{3}\right)$ 87.29-7.22 (m, 2H), 7.22-7.11 (m, 3H), $6.90(\mathrm{~s}, 1 \mathrm{H}), 3.49(\mathrm{~s}$, 1H), $3.34(\mathrm{~s}, 1 \mathrm{H}), 2.62-2.58(\mathrm{~m}, 2 \mathrm{H}), 1.93-1.85(\mathrm{~m}, 5 \mathrm{H}) . \mathrm{HRMS}[\mathrm{M}+\mathrm{H}]^{+}:$230.1113, found, 230.1103. 
3.1.18. N-phenylpropyl-3-phenylmaleimide (18)

Yield 70.4\%. ${ }^{1} \mathrm{H}$ NMR (500 MHz, $\left.\mathrm{CDCl}_{3}\right) \delta 7.50-7.43(\mathrm{~m}, 3 \mathrm{H}), 7.39(\mathrm{t}, \mathrm{J}=5.7 \mathrm{~Hz}, 3 \mathrm{H}), 7.26-7.17(\mathrm{~m}$, 2H), 7.17-7.07 (m, 3H), $3.57(\mathrm{~s}, 1 \mathrm{H}), 3.45(\mathrm{~s}, 1 \mathrm{H}), 2.58-2.54(\mathrm{~m}, 2 \mathrm{H}), 2.00-1.86(\mathrm{~m}, 2 \mathrm{H}) . \mathrm{HRMS}[\mathrm{M}+\mathrm{H}]^{+}$: 292.1267, found, 292.1259 .

\subsubsection{N-phenylpropyl-3,4-dimethylmaleimide (19)}

Yield 72.6\%. ${ }^{1} \mathrm{H}$ NMR (500 MHz, $\left.\mathrm{CDCl}_{3}\right)$ 87.30-7.24 (m, 2H), 7.24-7.12 (m, 3H), 3.34-3.30 (m, 2H), 2.64-2.60 (m, 2H), 1.97-1.88 (m, 2H), 1.87-1.83 (m, 6H). HRMS [M + H] $]^{+}$: 244.1263, found, 244.1259.

3.1.20. N-phenylpropyl-3,4-dichloromaleimide (20)

Yield 72.5\%. ${ }^{1} \mathrm{H}$ NMR (500 MHz, $\left.\mathrm{CDCl}_{3}\right)$ 87.28-7.22 (m, 1H), 7.22-7.12 (m, 2H), 3.44-3.40 (m, 1H), 2.62-2.58 (m, 1H), 1.92-1.88 (m, 1H). HRMS [M + H] $]^{+}: 284.0174$, found, 284.0167.

\subsection{General Synthetic Procedure for Maleimides 21-35}

In the first step, maleic anhydride $(1.96 \mathrm{~g}, 0.02 \mathrm{~mol})$ with $10 \mathrm{~mL}$ of acetic acid was added to a three-necked flask. At the same time, 4-fluoroaniline $(1.89 \mathrm{~mL}, 0.02 \mathrm{~mol})$ with $15 \mathrm{~mL}$ acetic acid was dripped into the three-necked flask through a constant-pressure dropping funnel at room temperature for $30 \mathrm{~min}$. For the second step, the reaction mixture was heated to $120^{\circ} \mathrm{C}$ and refluxed for $2-3 \mathrm{~h}$. Then, the concentrated solution was obtained by rotary evaporation after the reaction solution was cooled to room temperature. The concentrated solution was isolated by silica gel column to obtain the target compound 21. Other maleimide derivatives were synthesized similarly. The physical constants and spectral data of compounds 21-35 are summarized below.

3.2.1. $N$-(4-fluorophenyl)-maleimide (21)

Yield 83.8\%. ${ }^{1} \mathrm{H}$ NMR (500 MHz, $\left.\mathrm{CDCl}_{3}\right)$ 87.36-7.32 (m, 2H), 7.21-7.14 (m, 2H), $6.88(\mathrm{q}, 2 \mathrm{H})$. HRMS $[\mathrm{M}+\mathrm{H}]^{+}:$192.0378, found, 192.0383 .

3.2.2. $N$-(4-fluorophenyl)-3-methylmaleimide (22)

Yield 84.7 \%. ${ }^{1} \mathrm{H}$ NMR (500 MHz, $\left.\mathrm{CDCl}_{3}\right)$ 87.36-7.32 (m, 2H), 7.18-7.14 (m, 2H), $6.50(\mathrm{q}, \mathrm{J}=1.7 \mathrm{~Hz}$, 1H), $1.92(\mathrm{~d}, \mathrm{~J}=1.8 \mathrm{~Hz}, 3 \mathrm{H})$. HRMS [M + H] ${ }^{+}:$206.0525, found, 206.0539.

\subsection{3. $N$-(4-fluorophenyl)-3-phenylmaleimide (23)}

Yield 77.2 \%. ${ }^{1} \mathrm{H}$ NMR (500 MHz, DMSO) $87.64-7.05$ (m, 6H), 3.59-3.56 (m, 2H), 3.35-3.26 (m, 2H). HRMS $[\mathrm{M}+\mathrm{H}]^{+}:$268.0687, found, 268.0696 .

3.2.4. N-(4-fluorophenyl)-3,4-dimethylmaleimide (24)

Yield 85.7 \%. ${ }^{1} \mathrm{H}$ NMR (500 MHz, $\left.\mathrm{CDCl}_{3}\right) \delta 7.45(\mathrm{~m}, 2 \mathrm{H}), 7.23(\mathrm{~m}, 2 \mathrm{H}), 2.72(\mathrm{~s}, 6 \mathrm{H}) . \mathrm{HRMS}[\mathrm{M}+\mathrm{H}]^{+}$: 220.0687, found, 220.0696 .

3.2.5. $N$-(4-fluorophenyl)-3,4-dichloromaleimide (25)

Yield 87.2 \%. ${ }^{1} \mathrm{H}$ NMR (500 MHz, $\left.\mathrm{CDCl}_{3}\right)$ 87.37-7.32 (m, 2H), 7.22-7.17(m, 2H). HRMS [M + H] ${ }^{+}$: 259.9612, found, 259.9603.

3.2.6. $N$-(4-chlorophenyl)-maleimide (26)

Yield 86.5\%. ${ }^{1} \mathrm{H}$ NMR (500 MHz, $\left.\mathrm{CDCl}_{3}\right)$ 87.48-7.44(m, 2H), 7.36-7.30 (m, 2H), $6.88(\mathrm{~m}, 2 \mathrm{H})$. HRMS $[\mathrm{M}+\mathrm{H}]^{+}:$208.0076, found, 208.0087. 
3.2.7. N-(4-chlorophenyl)-3-methylmaleimide (27)

Yield 83.5\%. ${ }^{1} \mathrm{H}$ NMR (500 MHz, $\left.\mathrm{CDCl}_{3}\right)$ 87.46-7.42 (m, 2H), 7.35-7.31 (m, 2H), $6.50(\mathrm{q}, \mathrm{J}=1.7 \mathrm{~Hz}$, $1 \mathrm{H}), 2.19(\mathrm{~d}, \mathrm{~J}=1.8 \mathrm{~Hz}, 3 \mathrm{H})$. HRMS $[\mathrm{M}+\mathrm{H}]^{+}:$222.0253, found, 222.0244.

3.2.8. $\mathrm{N}$-(4-chlorophenyl)-3-phenylmaleimide (28)

Yield 72.7\%. ${ }^{1} \mathrm{H}$ NMR (500 MHz, DMSO) $87.65-7.00(\mathrm{~m}, 6 \mathrm{H}), 3.69-3.51(\mathrm{~m}, 2 \mathrm{H}), 3.25-3.20(\mathrm{~m}, 2 \mathrm{H})$. HRMS $[\mathrm{M}+\mathrm{H}]^{+}:$284.0412, found, 284.0400 .

3.2.9. $\mathrm{N}$-(4-chlorophenyl)-3,4-dimethylmaleimide (29)

Yield 83.5\%. ${ }^{1} \mathrm{H}$ NMR (500 MHz, $\left.\mathrm{CDCl}_{3}\right) \delta 7.43(\mathrm{~m}, 2 \mathrm{H}), 7.34(\mathrm{~m}, 2 \mathrm{H}), 2.07(\mathrm{~s}, 6 \mathrm{H})$. HRMS [M + H] ${ }^{+}$: 236.0412, found, 236.0400 .

3.2.10. $\mathrm{N}$-(4-chlorophenyl)-3,4-dichloromaleimide (30)

Yield 82.7\%. ${ }^{1} \mathrm{H}$ NMR $\left(500 \mathrm{MHz}, \mathrm{CDCl}_{3}\right)$ 87.50-7.45 (m, 2H), 7.35-7.31 (m, 2H). HRMS [M + H] ${ }^{+}$: 275.9313, found, 275.9308 .

3.2.11. $\mathrm{N}$-(4-bromophenyl)-maleimide (31)

Yield 73.8\%. ${ }^{1} \mathrm{H}$ NMR (500 MHz, $\left.\mathrm{CDCl}_{3}\right)$ 87.62-7.53 (m, 1H), 7.53-7.49 (m, 1H), 7.46-7.31 (m, 1H). HRMS $[\mathrm{M}+\mathrm{H}]^{+}:$251.9586, found, 251.9582.

3.2.12. $\mathrm{N}$-(4-bromophenyl)-3-methylmaleimide (32)

Yield 69.5\%. ${ }^{1} \mathrm{H}$ NMR (500 MHz, $\left.\mathrm{CDCl}_{3}\right) \delta 7.62-7.48(\mathrm{~m}, 2 \mathrm{H}), 7.46-7.31(\mathrm{~m}, 2 \mathrm{H}), 6.96(\mathrm{~s}, 1 \mathrm{H})$, $1.93-1.89(\mathrm{~m}, 3 \mathrm{H})$. HRMS $[\mathrm{M}+\mathrm{H}]^{+}: 265.9742$, found, 265.9738.

3.2.13. $\mathrm{N}$-(4-bromophenyl)-3-phenylmaleimide (33)

Yield 77.7\%. ${ }^{1} \mathrm{H}$ NMR $\left(500 \mathrm{MHz}, \mathrm{CDCl}_{3}\right) 87.60-7.50(\mathrm{~m}, 11 \mathrm{H}), 7.50-7.38(\mathrm{~m}, 32 \mathrm{H}), 7.38(\mathrm{~d}, \mathrm{~J}=0.7 \mathrm{~Hz}$, 1H), 7.37-7.28 (m, 11H). HRMS [M + H] $]^{+}$: 327.9892, found, 327.9895.

3.2.14. $\mathrm{N}$-(4-bromophenyl)-3,4-dimethylmaleimide (34)

Yield 74.1\%. ${ }^{1} \mathrm{H}$ NMR (500 MHz, $\left.\mathrm{CDCl}_{3}\right) \delta 7.64-7.49(\mathrm{~m}, 1 \mathrm{H}), 7.42-7.28(\mathrm{~m}, 1 \mathrm{H}), 1.84-1.80(\mathrm{~m}, 3 \mathrm{H})$. HRMS $[\mathrm{M}+\mathrm{H}]^{+}:$279.9886, found, 279.9895 .

\subsubsection{N-(4-bromophenyl)-3,4-dichloromaleimide (35)}

Yield 83.2\%. ${ }^{1} \mathrm{H}$ NMR $\left(500 \mathrm{MHz}, \mathrm{CDCl}_{3}\right)$ 87.64-7.49 (m, 1H), 7.41-7.27 (m, 1H). HRMS [M + H] ${ }^{+}$: 319.8811, found, 319.8802 .

\subsection{Biological Activity}

\subsubsection{Chitin Synthase Inhibitory Activity}

The CHS inhibitory activities of all synthesized compounds 1-35 were evaluated in comparison with that of commercially available polyoxin $B$ in vitro. The results showed that the inhibitory rate of CHS of 14 compounds compared to the semi-inhibitory concentration of CHS by polyoxin B $\left(\mathrm{IC}_{50}=0.19 \mathrm{mM}\right)$ were over $50 \%$. Therefore, the $\mathrm{IC}_{50}$ values of these 14 compounds on CHS were studied and summarized in Table 2. 
Table 2. The $\mathrm{IC}_{50}$ values of different maleimides against $\mathrm{CHS}$.

\begin{tabular}{cccccc}
\hline Compound & $\mathrm{IC}_{\mathbf{5 0}}(\mathbf{m M})$ & Compound & $\mathrm{IC}_{\mathbf{5 0}}(\mathbf{m M})$ & Compound & $\mathrm{IC}_{\mathbf{5 0}}(\mathbf{m M})$ \\
\hline $\mathbf{4}$ & 0.26 & $\mathbf{2 0}$ & 0.12 & $\mathbf{3 1}$ & 0.29 \\
$\mathbf{6}$ & 0.26 & $\mathbf{2 1}$ & 0.34 & $\mathbf{3 2}$ & 0.37 \\
$\mathbf{7}$ & 0.32 & $\mathbf{2 4}$ & 0.38 & $\mathbf{3 3}$ & 0.30 \\
$\mathbf{1 1}$ & 0.21 & $\mathbf{2 6}$ & 0.17 & $\mathbf{3 5}$ & 0.23 \\
$\mathbf{1 9}$ & 0.20 & $\mathbf{3 0}$ & 0.23 & Polyoxin B & 0.19 \\
\hline
\end{tabular}

\subsubsection{In Vitro Antifungal Activity}

The antifungal activities of all target compounds 1-35 were evaluated in vitro against S. sclerotiorum using the mycelium growth rate method in glassware. The clinical antifungal drugs pyrimethanil and polyoxin B were used as positive controls. The $\mathrm{EC}_{50}$ values of these target compounds on $\mathrm{S}$. sclerotiorum in vitro are summarized in Table 3, and compound 20, which had the best inhibitory effect on CHS among the 35 tested compounds, had an inhibitory effect on the growth of $S$. sclerotiorum mycelial at different concentrations as shown in Figure 3.

Table 3. The $\mathrm{EC}_{50}$ values of different maleimides against S.sclerotiorum.

\begin{tabular}{cccccc}
\hline Compound & EC $_{\mathbf{5 0}}(\mu \mathrm{g} / \mathbf{m L})$ & Compound & $\mathrm{EC}_{\mathbf{5 0}}(\boldsymbol{\mu g} / \mathbf{m L})$ & Compound & EC $_{\mathbf{5 0}}(\mu \mathrm{g} / \mathbf{m L})$ \\
\hline $\mathbf{1}$ & 15.82 & $\mathbf{1 4}$ & 17.92 & $\mathbf{2 7}$ & 75.83 \\
$\mathbf{2}$ & 28.95 & $\mathbf{1 5}$ & 145.43 & $\mathbf{2 8}$ & 52.27 \\
$\mathbf{3}$ & 30.17 & $\mathbf{1 6}$ & 15.08 & $\mathbf{2 9}$ & 72.95 \\
$\mathbf{4}$ & 12.73 & $\mathbf{1 7}$ & 54.64 & $\mathbf{3 0}$ & 19.95 \\
$\mathbf{5}$ & 48.47 & $\mathbf{1 8}$ & 39.95 & $\mathbf{3 1}$ & 5.78 \\
$\mathbf{6}$ & 6.95 & $\mathbf{1 9}$ & 9.13 & $\mathbf{3 2}$ & 7.83 \\
$\mathbf{7}$ & 28.65 & $\mathbf{2 0}$ & 8.47 & $\mathbf{3 3}$ & 9.52 \\
$\mathbf{8}$ & 48.25 & $\mathbf{2 1}$ & 16.23 & $\mathbf{3 4}$ & 42.13 \\
$\mathbf{9}$ & 14.59 & $\mathbf{2 2}$ & 112.35 & $\mathbf{3 5}$ & 11.28 \\
$\mathbf{1 0}$ & 31.07 & $\mathbf{2 3}$ & 195.68 & Pyrimethanil & 5.62 \\
$\mathbf{1 1}$ & 9.23 & $\mathbf{2 4}$ & 75.38 & Polyoxin B & 8.95 \\
$\mathbf{1 2}$ & 45.38 & $\mathbf{2 5}$ & 25.92 & & \\
$\mathbf{1 3}$ & 37.63 & $\mathbf{2 6}$ & 13.25 & & \\
\hline
\end{tabular}
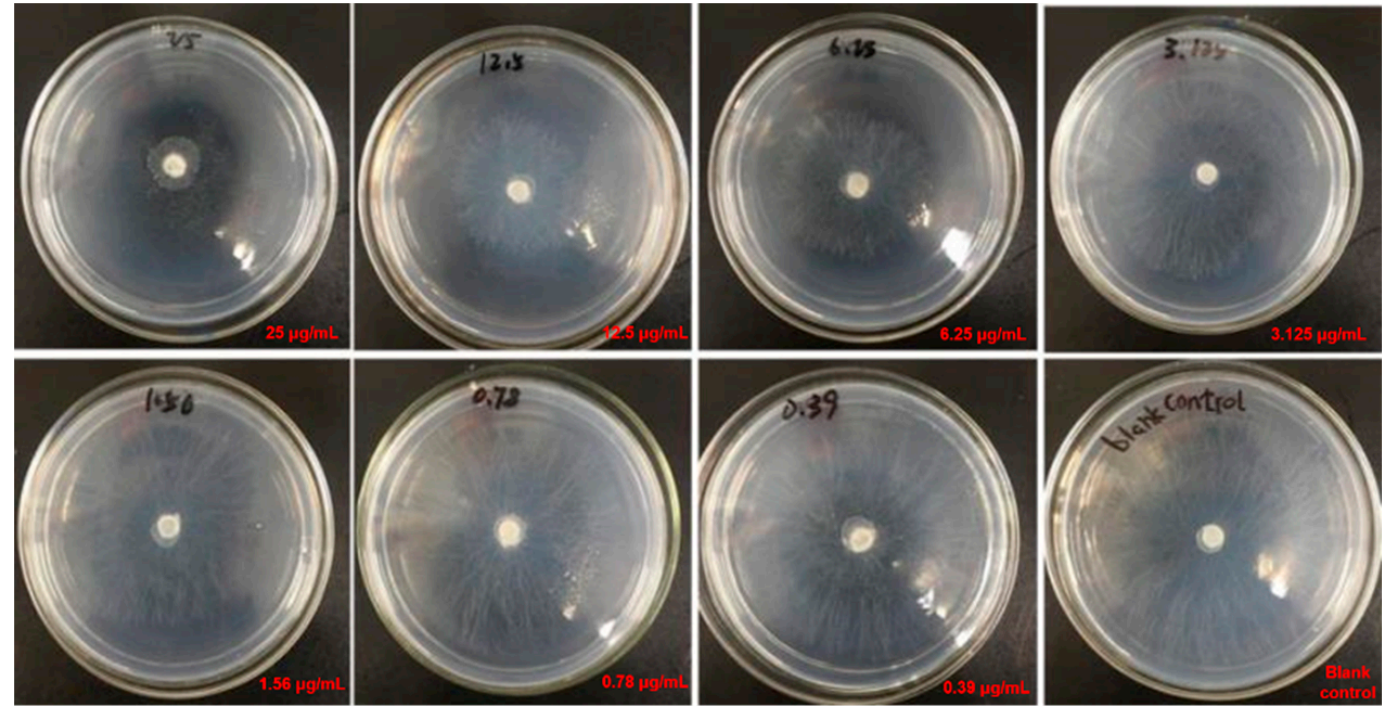

Figure 3. The inhibitory effect of compound 20 on S. sclerotiorum mycelial at 25, 12.5, 6.25, 3.125, 1.56, $0.78,0.39 \mu \mathrm{g} / \mathrm{mL}$ and blank control, which were incubated at $23^{\circ} \mathrm{C}$ for $36 \mathrm{~h}$. 


\section{Discussion}

The $\mathrm{IC}_{50}$ values in Table 2 revealed that nearly half of the compounds displayed moderate to high inhibitory activities on CHS. Compound 20 displayed highest CHS inhibitory activity $\left(\mathrm{IC}_{50}=0.12 \mathrm{mM}\right)$ among all the tested compounds, which was more effective than polyoxin $\mathrm{B}$, which acts as control drug with an $\mathrm{IC}_{50}$ of $0.19 \mathrm{mM}$. Compounds $11,19,26,30$ and 35 had $\mathrm{IC}_{50}$ values of $0.21,0.20,0.17,0.23$, $0.23 \mathrm{mM}$, respectively, which were almost equal to that of polyoxin B. Compounds $4,6,31$ and 33 had slightly lower CHS inhibitory activity, while compounds 7, 21, 24 and 33 exerted the lowest inhibition potency among these compounds. Generally, the number of carbon bonds between the five-membered ring and the benzene ring $(n=3)$ was a key factor that determined the most potent inhibitory activity toward CHS, such as for compounds 19 and 20.

As chitin is essential for fungi survival, we guessed that the design of these CHS inhibitors may inhibit fungi growth, because they can reduce the synthesis of chitin by inhibiting the activity of CHS. The results of their antifungal activity in Table 3 showed that the target compounds 1-35 exhibited moderate to excellent antifungal efficacy against $S$. sclerotiorum. Compounds 6, 19, 20, 31 and 32 displayed $\mathrm{EC}_{50}$ values of $6.95,9.13,8.47,5.78,7.83 \mu \mathrm{g} / \mathrm{mL}$, respectively, having better inhibitory activities than polyoxin B. Their inhibitory activities were almost equal to pyrimethanil, especially 31 with an $\mathrm{EC}_{50}$ value of $5.78 \mu \mathrm{g} / \mathrm{mL}$. Therefore, these five compounds displayed favorable antifungal activities in comparison to pyrimethanil and polyoxin B, which indicated that they have a higher potential as a superactive fungicide.

This data shows that the design of these compounds to act as antifungal agents was reasonable, and superactivity towards $\mathrm{CHS}$ and bioactivity against $S$. sclerotiorum can be seen. As a rule, some compounds which displayed superior inhibitory activity against CHS showed excellent antifungal activity.

\section{Conclusions}

CHS, an attractive and green target for new fungicides, was inhibited by some biologically active compounds. In this study, a series of maleimide compounds were synthesized and researched for their anti-CHS and antifungal activity. The anti-CHS assay data indicated that most of the target compounds displayed moderate to excellent activity against CHS; in particular, compound $\mathbf{2 0}$ was the best CHS inhibitor among the 35 target compounds, with an $\mathrm{IC}_{50}$ value of $0.12 \mathrm{mM}$. The antifungal assay results indicated that all maleimide compounds had varying degrees of inhibition towards S. sclerotiorum. Among them, compounds 6, 19, 20, 31 and 32 displayed excellent antifungal activity, which were almost equal to the standard drugs pyrimethanil and polyoxin B. Furthermore, compounds with three carbon bonds between the five-membered ring and the benzene ring had higher anti-CHS and antifungal activity than other target compounds. This indicated that it is possible to develop new CHS inhibitors from these target compounds, which may have potential for the treatment of fungal infections.

Author Contributions: X.C. and Y.L. conceived and designed the experiments; X.S. and S.Q. performed the experiments; Y.B. and H.C. analyzed the data; X.S. wrote the paper. All authors have read and agreed to the published version of the manuscript.

Funding: We are grateful for the research grants from the National Natural Science Foundation of China (No. 21572206 and No. 31601390).

Conflicts of Interest: The authors declare no conflict of interest.

\section{References}

1. Fisher, M.C.; Henk, D.A.; Briggs, C.J.; Brownstein, J.S.; Madoff, L.C.; McCraw, S.L.; Gurr, S.J. Emerging fungal threats to animal, plant and ecosystem health. Nature 2013, 484, 186-194. [CrossRef] [PubMed]

2. Zhu, S.P.; Wang, W.Y.; Fang, K.; Li, Z.G.; Dong, G.Q.; Miao, Z.Y.; Yao, J.Z.; Zhang, W.N.; Sheng, C.Q. Design, synthesis and antifungal activity of carbazole derivatives. Chin. Chem. Lett. 2014, 25, 229-233. [CrossRef]

3. Scheffler, R.J.; Colmer, S.; Tynan, H.; Demain, A.L.; Gullo, V.P. Antimicrobials, drug discovery, and genome mining. Appl. Microbiol. Biot. 2013, 97, 969-978. [CrossRef] 
4. Kapteyn, J.C.; Hoyer, L.L.; Hecht, J.E.; Müller, W.H.; Andel, A.; Verkleij, A.J.; Makarow, M.; Van Den Ende, H.; Klis, F.M. The cell wall architecture of candida lbicans wild-type cells and cell wall-defective mutants. Mol. Microbiol. 2000, 35, 601-611. [CrossRef] [PubMed]

5. Lenardon, M.D.; Munro, C.A.; Gow, N.A.R. Chitin synthesis and fungal pathogenesis. Curr. Opin. Microbiol. 2010, 13, 416-423. [CrossRef] [PubMed]

6. Isono, K.; Nagatsu, J.; Kawashima, Y.; Suzuki, S. Studies on polyoxins, antifungal antibiotics. J. Agric. Chem. Soc. Jpn. 1965, 29, 848-854.

7. Kobinata, K.; Uramoto, M.; Nishii, M.; Kusakabe, H.; Nakamura, G.; Isono, K. Neopolyoxins A, B, and C, New chitin synthetase inhibitors. Agric. Biol. Chem. 1980, 44, 1709-1711.

8. Uramoto, M.; Kobinata, K.; Jenkins, E.E.; McCloskey, J.A.; Higashijima, T.; Miyazawa, T. Neopolyoxins A, B, and C: New inhibitors of fungal cell wall chitin synthetase. Nucleic Acids Symp. Ser. 1980, 8, 69-71.

9. Shubitz, L.F.; Trinh, H.T.; Perrill, R.H.; Thompson, C.M.; Hanan, N.J.; Galgiani, J.N.; Nix, D.E. Modeling nikkomycin $\mathrm{Z}$ dosing and pharmacology in murine pulmonary coccidioidomycosis preparatory to phase 2 clinical trials. J. Infect. Dis. 2014, 209, 1949-1954. [CrossRef]

10. Jeong, T.S.; Hwang, E.I.; Lee, H.B.; Lee, E.S.; Kim, Y.K.; Min, B.S.; Bae, K.H.; Bok, S.H.; Kim, S.U. Chitin synthase II inhibitory activity of ursolic acid, isolated from crataegus pinnatifida. Planta Med. 1999, 65, 261-262. [CrossRef]

11. Kim, C.J.; Yoo, H.R.; Yoo, M.S.; Kwon, B.E.; Hwang, K.J. Attitude, beliefs, and intentions to care for sars patients among korean clinical nurses. Taehan Kanho Hakhoe Chi 2006, 36, 596-603. [PubMed]

12. Uda, J.; Obi, K.; Iwase, K.; Sugimoto, O.; Ebisu, H.; Matsuda, A. Synthesis and structure-activity relationships of novel nikkomycin analogs: Inhibitors of the fungal cell wall biosynthesis enzyme chitin synthase. Nucleic Acids Symp. Ser. 1999, 42, 13-14. [CrossRef] [PubMed]

13. Tokumura, T.; Horie, T. Kinetics of nikkomycin $\mathrm{Z}$ degradation in aqueous solution and in plasma. Biol. Pharm. Bull. 1997, 20, 577-580. [CrossRef] [PubMed]

14. Watanabe, S.; Igarashi, Y.; Yagami, K. Antimicrobial activity of some Narylalkyl maleimides. Pest. Sci. 2006, 34, 99-104. [CrossRef]

15. Wattanadilok, R.; Sawangwong, P.; Rodrigues, C.; Cidade, H.; Pinto, M.; Pinto, E.; Silva, A.; Kijjoa, A. Antifungal activity evaluation of the constituents of haliclona baeri and haliclona cymaeformis, collected from the gulf of thailand. Mar. Drugs 2007, 5, 40-51. [CrossRef]

16. Bradsher, C.K.; Harvan, D.J. Stereochemistry of the addition of $N$-arylmaleimides to the acridizinium ion. J. Org. Chem. 1971, 36, 67-70. [CrossRef]

17. Uehara, Y.I.; Fisher, J.M.; Rabinovitz, M. Showdomycin and its reactive moiety, maleimide. A comparison in selective toxicity and mechanism of action in vitro. Biochem. Pharmacol. 1980, 29, 2199-2204. [CrossRef]

18. Kar, S.; Wang, M.F.; Yao, W.; Michejda, C.J.; Carr, B.I. PM-20, a novel inhibitor of Cdc25A, induces extracellular signal-regulated kinase $1 / 2$ phosphorylation and inhibits hepatocellular carcinoma growth in vitro and in vivo. Mol. Cancer Ther. 2006, 5, 1511-1519. [CrossRef]

19. Hwang, E.I.; Lee, Y.M.; Lee, S.M.; Yeo, W.H.; Moon, J.S.; Kang, T.H.; Park, K.D.; Kim, S.U. Inhibition of chitin synthase 2 and antifungal activity of lignans from the stem bark of Lindera erythrocarpa. Planta Med. 2007, 73, 679-682. [CrossRef]

20. Gholap, A.R.; Toti, K.S.; Shirazi, F.; Kumari, R.; Bhat, M.K.; Deshpande, M.V.; Srinivasan, K.V. Synthesis and evaluation of antifungal properties of a series of the novel 2-amino-5-oxo-4-phenyl-5,6,7,8tetrahydroquinoline-3-carbonitrile and its analogues. Bioorg. Med. Chem. 2007, 15, 6705-6715. [CrossRef]

21. Chen, X.L.; Zhu, X.H.; Ding, Y.C.; Shen, Y.C. Antifungal activity of tautomycin and related compounds against Sclerotinia sclerotiorum. J. Antibiot. 2011, 64, 563-569. [CrossRef] [PubMed]

22. Chen, X.L.; Zheng, Y.G.; Shen, Y.C. Natural products with maleic anhydride structure: Nonadrides, tautomycin, chaetomellic anhydride, and other compounds. Chem. Rev. 2007, 107, 1777-1830. [CrossRef] [PubMed]

23. Li, W.; Fan, Y.X.; Shen, Z.Z.; Shen, Y.C.; Chen, X.L. Antifungal activity of simple compounds with maleic anhydride or dimethylmaleimide structure against botrytis cinerea. J. Pestic. Sci. 2012, 37, 247-251. [CrossRef]

24. Shen, Z.Z.; Fan, Y.X.; Li, F.G.; Chen, X.L.; Shen, Y.C. Synthesis of N-substituted dimethylmaleimides and their antifungal activities against Sclerotinia sclerotiorum. J. Pest. Sci. 2013, 86, 353-360. [CrossRef] 
25. Ke, S.Y.; Liu, F.Y.; Wang, N.; Yang, Q.; Qian, X.H. 1,3,4-Oxadiazoline derivatives as novel potential inhibitors targeting chitin biosynthesis: Design, synthesis and biological evaluation. Bioorg. Med. Chem. Lett. 2009, 19, 332-335. [CrossRef] [PubMed]

26. Li, C.F.; Wang, F.; Ma, H.; Luo, M.; Yu, Y.H. Study on antibacterial activity of Arnebia euchroma (Royle) johnst hairy roots extract. J. Agric. Univ. Hebei 2010, 33, 92-96.

27. Alexander, B.; Browse, D.J.; Reading, S.J.; Benjamin, I.S. A simple and accurate mathematical method for calculation of the EC 50 . J. Pharmacol. Toxicol. Meth. 1999, 41, 55-58. [CrossRef]

2020 by the authors. Licensee MDPI, Basel, Switzerland. This article is an open access article distributed under the terms and conditions of the Creative Commons Attribution (CC BY) license (http://creativecommons.org/licenses/by/4.0/). 